\title{
CONECTA-2: entrenamiento de la cognición social y el lenguaje, al servicio de la eficacia comunicativa. Una experiencia clínica interactiva con personas con diagnóstico de esquizofrenia CONECTA-2: training of social cognition and language, at service of communicative effectiveness. A clinical and interactive experience with people with diagnosis of schizophrenia
}

Alicia Figueroa B. ${ }^{1}$, Sonia Oyarzún S. ${ }^{2}$ y Eduardo Durán L. ${ }^{3}$

\begin{abstract}
Introduction: In this paper, we propose to describe a pilot therapeutic experience with a program developed by the team, which we call CONECTA-2, a training plan for social cognition and communication skills for people diagnosed with schizophrenia. Our objective was to establish whether a theoretical-practical therapeutic clinical device, training in executive functions, processes of social cognition and language, can improve the effectiveness of communication in people diagnosed with schizophrenia. Method: Participants were 10 people between 18 and 24 years old, 7 men and 3 women, diagnosed with schizophrenia for less than three years at the time of the program. A prior and subsequent evaluation of emotional attribution and communicative effectiveness was carried out. 24 group sessions were held, training in executive functions, social cognition and pragmatic-communicative skills. Results: Positive changes were verified both in emotional attribution and in the communicative effectiveness of the participants. Conclusions: The results of this pilot experience present a challenge in terms of developing clinical intervention plans that allow rehabilitating those skills to achieve a better adaptation and social insertion of people with schizophrenia.
\end{abstract}

Key words: Schizophrenia, language, cognition, communication. Rev Chil Neuro-Psiquiat 2019; 57 (1): 9-18

Recibido: 23/03/2018

Aceptado: 28/03/2019

Los autores no presentan ningún tipo de conflicto de interés.

1 PhD Lingüística Clínica, Universidad de Chile, Facultad de Medicina, Departamento de Psiquiatría y Salud Mental Campus Sur, Unidad de Psicolingüística Clínica-Laboratorio de Psiquiatría Traslacional del BNI (Biomedical Neuroscience Institute).

2 Ps. Mg. Psicología Clínica. Universidad de Chile, Facultad de Medicina, Departamento de Psiquiatría y Salud Mental Campus Sur, Unidad de Psicolingüística Clínica.

3 MD. Mg. Psiquiatra, Universidad de Chile, Facultad de Medicina, Departamento de Psiquiatría y Salud Mental Campus Sur, Unidad de Psicolingüística Clínica. 
"La parte más importante de mi ambiente es mi prójimo. La conciencia de su actitud hacia mí es la percepción que normalmente abre la mayoría de mis vergüenzas, indignaciones y miedos".

(William James, 1884).

\section{Introducción}

$\mathrm{D}$ entro de las más complejas facultades humanas, la comunicación se sitúa como nuestro soporte intersubjetivo como ejemplo paradigmático. La eficacia comunicativa redundará, inequívocamente, en mayores posibilidades de éxito social para el individuo. De esta forma, esta habilidad se convierte en una manifestación social que cataliza la inclusión o exclusión de los individuos.

La comunicación, exige la habilidad de los hablantes para determinar, evaluar y comprender indicios y claves sociales que le permitan una participación dinámica y eficiente en el medio en el que está inserto (Couture, 2006). Esta capacidad para extraer y decodificar claves intersubjetivas, depende a su vez, de un sinnúmero de procesos, entre los que destacan la capacidad de atribución emocional, empatía, teoría de la mente, interpretación de claves contextuales, todas enmarcadas en el constructo cognición social, que abarca una multiplicidad de dimensiones cognitivo-afectivo-sociales, que demandan una comprensión compleja e integrada del ser humano.

Para Adolphs (2001), la cognición social se define como "la capacidad de construir representaciones de la relación entre uno mismo y los demás, y utilizar esas representaciones de manera flexible para guiar el comportamiento social".

La cognición social puede afectar el resultado de habilidades de la vida cotidiana, dado que se requiere una evaluación precisa de las señales del entorno para poder desempeñarse socialmente de manera adaptativa (Couture, 2006). Debido a esto, cuando no se cuenta con estos recursos, la capacidad de interactuar de manera eficaz, obviamente se verá restringida.
Para Vygotsky (1988), el hombre es producto de los cambios biológicos de la evolución de la especie y de desarrollo histórico-cultural de la sociedad. Filogenéticamente se han ido desarrollando en el ser humano importantes cambios biológicos, a nivel del sistema nervioso, o en el aparato fonoarticulatorio, que posibilitan que los procesos comunicativos hayan ido evolucionando a lo largo de la historia, los contextos culturales y los requerimientos sociales específicos.

De la misma forma, ontogenéticamente, la biología va permitiendo instauración de estructuras y redes que van posibilitando el desarrollo de funciones que dependen en gran medida de nuestra interacción con otros. Son la atención, y la intención conjunta, que se da en un vínculo afectivo primario, las que permiten que evolucione la capacidad de simbolizar, de mentalizar y de representarnos el mundo; y por otra parte, es el desarrollo de funciones como la capacidad de anticipación, memoria operativa, control cognitivo y funciones analítico-reflexivas, las que nos permiten una representación mental integrada y posibilitan el desarrollo de una estructura identitaria unificada y coherente, con un estilo de vinculación propio, capaz de interactuar con otros.

En personas que presentan patologías como el autismo o la esquizofrenia, se verifica una significativa perturbación en el modo en el cual se procesan los estímulos socio-contextuales, lo que impacta negativamente en la calidad de sus interacciones.

Subyacen a la cognición social, una serie de operaciones y funciones cognitivas que sustentan el comportamiento social.

Las personas diagnosticadas con esquizofrenia pueden mostrar déficits cognitivos en diferentes ámbitos, incluida la cognición social, presentando además dificultades en la capacidad atencional, memoria verbal, o funciones ejecutivas, y una significativa alteración en el reconocimiento emocional (Hall et al., 2004). Esta dificultad inevitablemente se traduce en una importante disfunción social (Emery et al., 2001).

Uno de los cuadros que más se ha estudiado en términos de la cognición social, es el autismo. Baron-Cohen (1989a, 1989b, 1991, 1992, 1993) 
Baron-Cohen, Leslie and Frith $(1985,1986)$ y Leslie y Frith $(1988,1990)$ han propuesto que hay una dificultad en la comprensión de los estados mentales de sí mismo y de los demás, que restringiría la comprensión de muchas situaciones sociales cotidianas. Yirmiya, Sigman, Kasari y Mundy (1992) propusieron un modelo centrado en los déficits de logro de la atención conjunta como el deterioro fundamental en personas diagnosticadas con autismo.

En esa misma línea, la esquizofrenia también se presenta como uno de los trastornos mentales en las que el mayor compromiso mórbido del sujeto se refleja en la afectación de las funciones cognitivo-sociales. De ello se desprende que subyace a esta patología un déficit en cognición social, en la metarrepresentación y, por tanto, en la toma de perspectiva, afectándose la forma de realizar representaciones simbólicas de sí mismo y del otro. La búsqueda de las bases de esta disfunción, han dado pie a un sinnúmero de estudios y teorizaciones, respecto a si la afectación central se encuentra a nivel afectivo-vincular o a nivel cognitivo, y de qué manera se impacta la instauración, consolidación o se deteriora la dimensión intersubjetiva.

El proyecto MATRICS: Measurement and Treatment Research to Improve Cognition un Schizophrenia, (Green y Nuechterlein, 2004), reconoce explícitamente el significativo rol de la cognición social en las personas con esta patología, al incluirlo dentro de las siete dimensiones cognitivas que se encuentran afectadas: Velocidad de procesamiento, atención, memoria de trabajo, aprendizaje verbal, aprendizaje visual, razonamiento y resolución de problemas y cognición social.

Este proyecto considera 5 dominios de la cognición social: Procesamiento emocional, teoría de la mente, percepción social, conocimiento social y estilo atribucional.

Se ha planteado que la cognición social podría predecir mejor la funcionalidad de la persona afectada con esquizofrenia, que los factores neurocognitivos o incluso que los síntomas clínicos ( $\mathrm{Pi}$ jnenborg, 2009; Fett, 2011). La Teoría de la Mente es la dimensión de la cognición social que más se asocia con el funcionamiento social (Fett, 2011), ejerciendo un rol de mediación entre la neurocognición y la funcionalidad (Schmidt, 2011).

Se han desarrollado numerosos programas de intervención en la cognición social para personas con esquizofrenia, como el TAR (Training of Affect Recognition), de Frommann, Streit y Wolwer, 2003, que se focaliza en el reconocimiento de emociones; el Programa de Percepción Social del IPT (Terapia Psicológica Integrada), de Roder y Brenner (2007), que se centra en la percepción social; el SCIT (Social Cognition and Interaction Training), de Penn, 2005); y el INT (Integrative Neurocognitive Therapy) de Roder et al., 2007). Todos ellos han demostrado eficacia clínica en diversas áreas de la cognición social, posicionándose como herramientas terapéuticas valiosas. $\mathrm{Al}$ intentar desarrollar dispositivos que puedan aportar en una mejor calidad de vida de las personas afectadas por esquizofrenia, considerando los déficits cognitivos sociales y no sociales que ellos presentan, surge la idea de desarrollar un programa que pueda ser utilizado en la clínica, y que permita integrar diferentes aspectos del funcionamiento que, finalmente, redundan en una mejor capacidad adaptativa. Poder integrar en un programa de entrenamiento clínico y grupal interactivo, los aspectos neurocognitivos, psicolingüísticos y de cognición social que subyacen a las habilidades comunicativas, nos puede permitir desarrollar y estimular capacidades necesarias para una mejor adaptación social que pueda aportar en una mejor inserción y un menor estigma.

\section{Caracterización de los participantes}

Los participantes de esta experiencia fueron un grupo de 10 personas con diagnóstico de esquizofrenia. Se consideraron personas con ese diagnóstico desde hace menos de tres años al momento del estudio. El rango de edad estuvo entre 18 y 24 años, 7 hombres y 3 mujeres (Tabla 1).

\section{Criterios de inclusión}

I. Confirmación de diagnóstico por al menos dos especialistas psiquiatras. 
Tabla 1. Caracterización de los participantes

\begin{tabular}{|ll|}
\hline $\mathrm{n}=$ & 10 \\
\hline Diagnóstico & Esquizofrenia \\
\hline Estadio de la enfermedad & Primer episodio \\
$\begin{array}{l}\text { Sexo } \\
\quad \text { Mujeres }\end{array}$ \\
$\quad$ Hombres & 3 \\
Subtipo de la enfermedad & 7 \\
\hline Edad & Paranoide \\
Escolaridad & 18 a 24 años \\
\hline
\end{tabular}

II. Consentimiento informado.

III. Administración previa de PANSS y evaluación por médico psiquiatra que certificó ausencia de sintomatología positiva y estabilización psicopatológica.

IV. Mayores de edad.

V. Hablantes nativos de español.

VI. Nivel intelectual normal.

\section{Criterios de exclusión}

I. Consumo de sustancias neurotóxicas.

II. Patología neurológica.

III. Intervención previa de estimulación neurocognitiva o cognición social.

IV. Tratamiento de terapia electroconvulsiva.

V. Descompensación psicopatológica.

\section{Esquema operativo}

El esquema operativo del programa CONECTA-2 comprendió una primera etapa de evaluación diagnóstica, aplicación del taller dividido en módulos temáticos y funcionales y evaluación postaller, como se aprecia en la Figura 1.

\section{Fase 1: Evaluación diagnóstica}

Diagnóstico de esquizofrenia

Se procuró asegurar el diagnóstico de esquizofrenia primer episodio, reclutando personas derivadas al Programa de Esquizofrenia GES.

\section{Nivel intelectual}

Se administró la Escala de Inteligencia de Wechsler para Adultos WAIS.

\section{Atribución emocional}

Eyes Test (Baron-Cohen, 2001): Este test se desarrolló basándose en conceptos de la teoría de la mente, considerando que personas sanas son capaces de determinar el estado mental de otros a partir de la expresión de la mirada. Se muestran 36 fotografías de la zona de los ojos de diferentes personas. Se debe elegir entre cuatro opciones escritas, la que represente mejor la expresión de la mirada presentada (Baron-Cohen, 2001; Burin D., Drake M. y Harris P., 2008).

Faux Pas: Se trata de 10 historias que contienen errores sociales o "metidas de patas" y 10 historias control, que evalúa la capacidad para detectar si una persona dice algo inadecuado, pudiendo afectar a otros, pero sin mala intención (Stone, V.E., Baron-Cohen, S. \& Knight, R.T, 1998).

\section{Habilidades pragmático-comunicativas}

Screening LEPSI, Lenguaje, Psicosis e Intersubjetividad. Screening LEPSI es un protocolo

Figura 1. Fases de

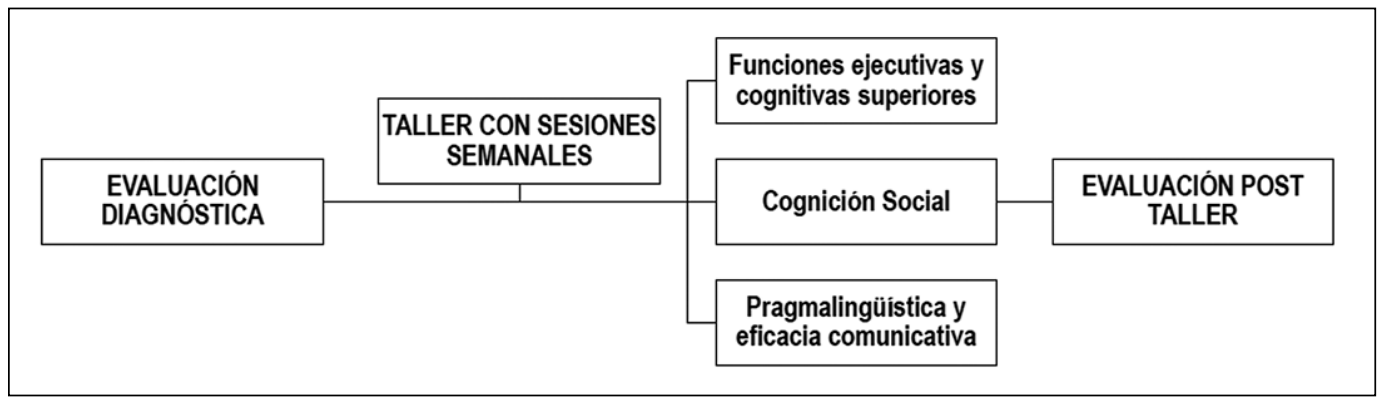


experimental para la valoración de categorías y dimensiones discursivas en términos de adecuación pragmática. Fue desarrollado por el equipo y se evaluó la fiabilidad de los índices con el juicio de 6 expertos de psiquiatría y lingüística. Su aplicación para CONECTA-2 se realizó como una evaluación test-retest.

Está dividido en tres partes organizadas como sigue:

Parte 1: Autoevaluación del paciente, incluye un cuestionario de 6 preguntas sobre la percepción que tiene sobre su desempeño comunicativo.

Parte 2: Análisis del discurso del paciente, se realiza una valoración en términos de niveles de adecuación pragmática de 37 categorías comunicativas agrupadas en cinco grupos para el análisis multidimensional del lenguaje: i. organización global pragmalinguística; ii. organización estructural (coherencia y cohesión); iii. ToM y pragmática (producción y comprensión); iv. coherencia interaccional (fluidez verbal y producción verbal) $\mathrm{y}$ aspectos paraverbales.

Parte 3: Cuestionario de 6 preguntas dirigido al terapeuta (profesional del equipo tratante de cada participante) para que evaluara la eficacia comunicativa del paciente.

\section{Fase 2: Taller}

Se organizó un taller de 2 sesiones semanales semiestructuradas de 1,5 h distribuidas en 24 módulos temáticos, que incluyeron las siguientes áreas de trabajo:
I. Funciones ejecutivas y cognitivas superiores: Se diseñaron 8 sesiones de estimulación cognitiva focalizadas en: atención sostenida, comprensión abstracta, planificación, memoria de trabajo verbal, organización, priorización, flexibilidad cognitiva. Las actividades se desarrollaron tanto de manera individual como grupal. En la Figura 2 se presentan algunos ejemplos de las tareas que se realizaron en este módulo.

\section{Cognición social}

Se diseñaron 8 sesiones de entrenamiento grupal en cognición social, las que se dividieron en módulos: reconocimiento y decodificación emocional, atribución emocional, teoría de la mente, empatía, toma de decisiones, claves contextuales.

Dentro del módulo de cognición social, se realizaron frecuentes ejercicios para explicitar el significado de expresiones físicas, en especial gestos faciales, para ello se confeccionaron materiales recogidos de diversos medios audiovisuales, principalmente de internet, de medios publicitarios y medios escritos, entre otros. Una de las consignas era descubrir la emoción representada y las claves gestuales que la ponían en evidencia. A pesar de que puede parecer sencillo de reconocer, con frecuencia presentaron juicios erróneos. En la Figura 3 presentamos una de las láminas que empleamos para este propósito:

Una variable de este ejercicio era una selección rápida de microexpresiones secuenciadas para una respuesta rápida. En la Figura 4 presentamos un ejemplo tomado de los materiales de Eckman:

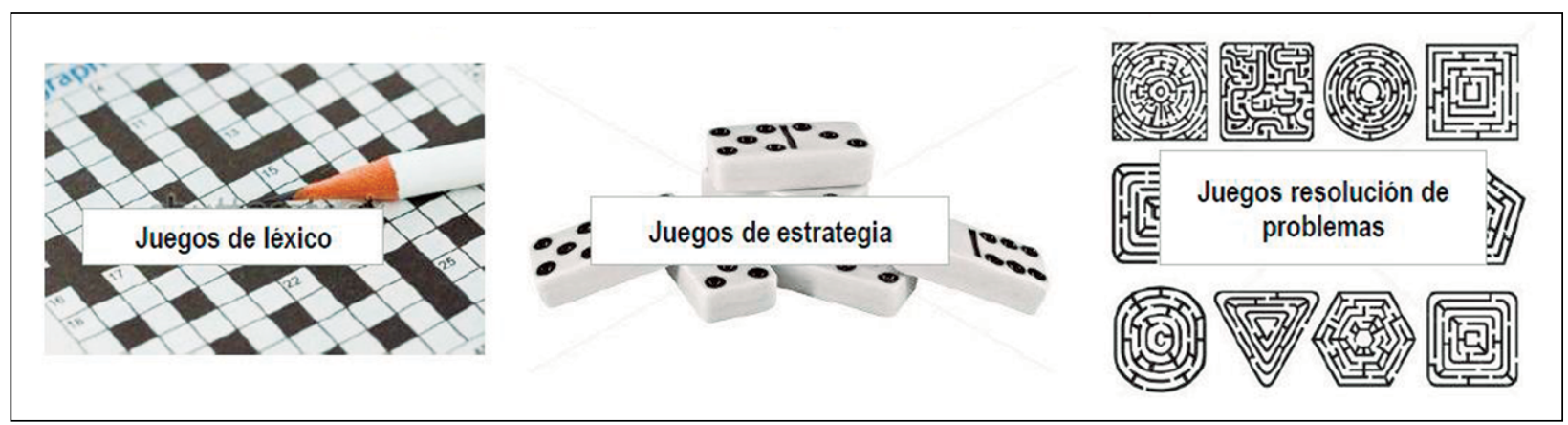

Figura 2. Tareas para estimular funciones ejecutivas y cognitivas superiores para estimular sistemas de memoria, planificación, toma de decisiones y resolución de problemas. 


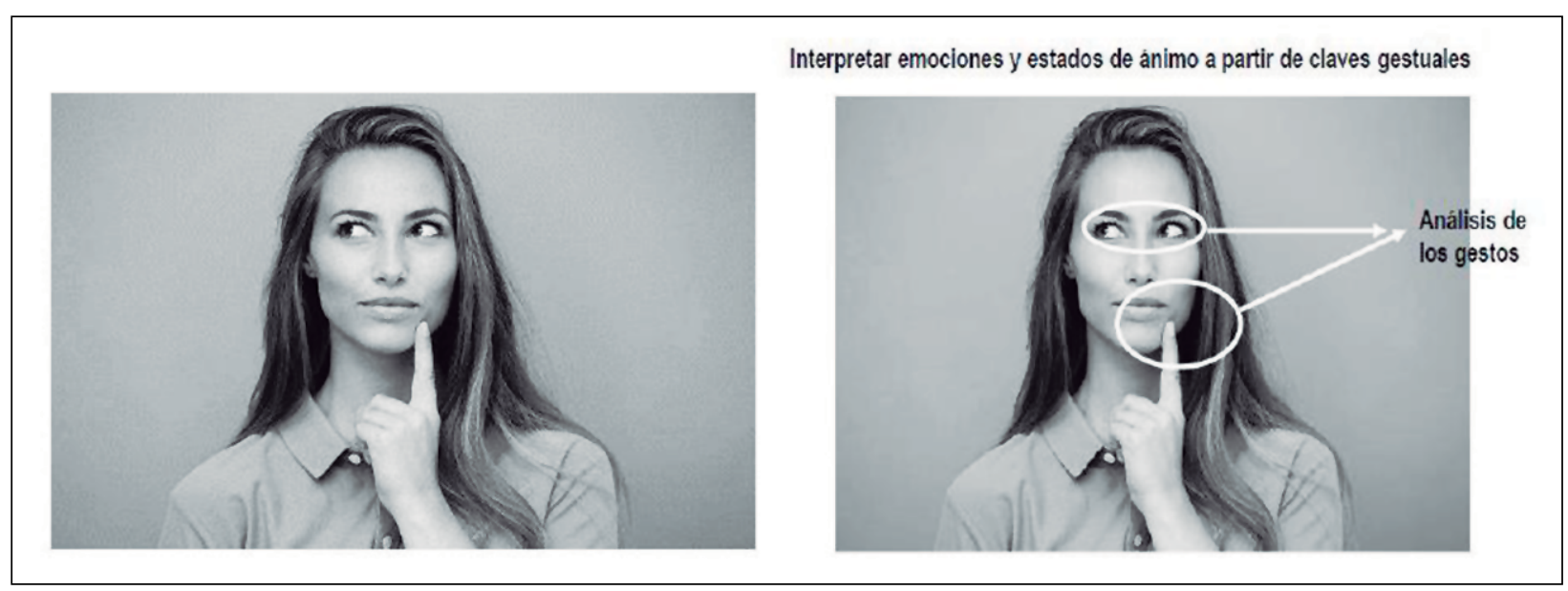

Figura 3. Análisis de los gestos faciales y de las emociones que representan.

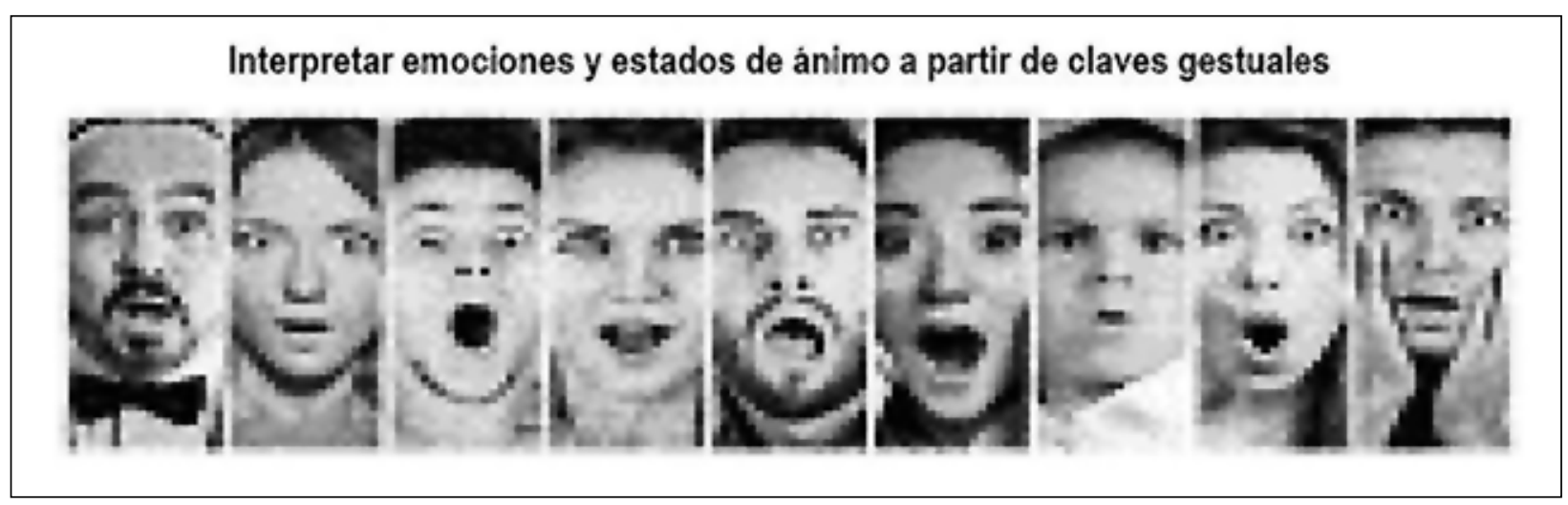

Figura 4. Identificación de las emociones y estados de ánimo.

\section{Pragmalingüística y eficacia comunicativa}

Se realizaron 16 sesiones de entrenamiento grupal práctico en habilidades pragmático-comunicativas para mejorar la eficacia comunicativa: gestualidad de las expresiones faciales y corporales, proxemia, negociación del significado de actos de habla, implicaturas y máximas conversacionales, situación y evento de habla, entrenamiento en la arquitectura conversacional, rutinas y estrategias conversacionales e interaccionales, entrenamiento en estrategias para mantener el tema central de la conversación, role playing y análisis de situaciones comunicativas diversas. En general se trabajaron estas habilidades en todo el programa, por tanto, sus límites funcionales de aplicación y entre- namiento superaron las 16 sesiones que aquí se describen.

La interpretación de los espacios proxémicos o de distancia interpersonal fue una actividad que se integró en este módulo dadas los frecuentes errores que los pacientes presentaban en la interacción. Se realizaron ejercicios para explicitar los límites de dichos espacios con el fin de identificar cuáles eran adecuados para interactuar con otras personas. En estos ejercicios se consideraron situaciones sociales comunes, como saludos, conversaciones, peticiones de información a un desconocido, etc. Se revisaron spot publicitarios y películas para analizar el comportamiento proxémico de los participantes y se aplicaron diferentes juegos de roles para 
ejercitar. La consigna era elegir al azar un número que correspondía a una situación comunicativa $\mathrm{X}$, luego se analizaba o se representaba entre los participantes. En la Figura 5 mostramos un esquema básico con el que se trabajaron los juegos de roles según las situaciones comunicativas que se iban escogiendo al azar.

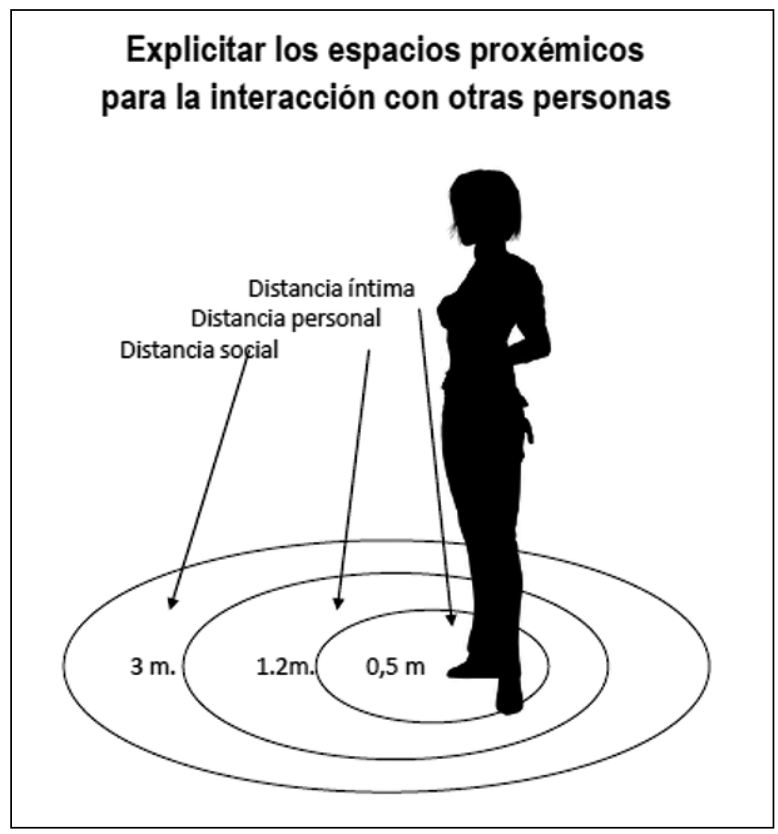

Figura 5. Espacios proxémicos.
Las tareas pragmalingüísticas se desarrollaron en forma transversal en el transcurso de todo el taller. Con frecuencia se les proponían situaciones que debían ser abordadas desde el discurso y teniendo en cuenta la lectura de los contextos situacionales, en todos estos casos también se trabajaron las habilidades comunicativas no verbales. En la Figura 6, algunos ejemplos de las situaciones discursivas que se plantearon para ejercitar.

\section{Fase 3: Evaluación}

Se administró el Test de las Miradas y el screening LEPSI al finalizar el Taller, realizándose una comparación pre y postaller. En ambos instrumentos se comprobó un incremento en los puntajes de entrada.

\section{Resultados}

Se verificó un aumento en las puntaciones de las pruebas en 8 de los 10 participantes, mientras que solamente en uno de ellos se mantuvo el puntaje de entrada al taller. Como puede observarse en la Tabla 2, en el primer Eyes test, los participantes presentaron puntuaciones similares; el incremento de los puntajes después del taller reflejó resultados diversos, en tres pacientes se consiguió un aumento

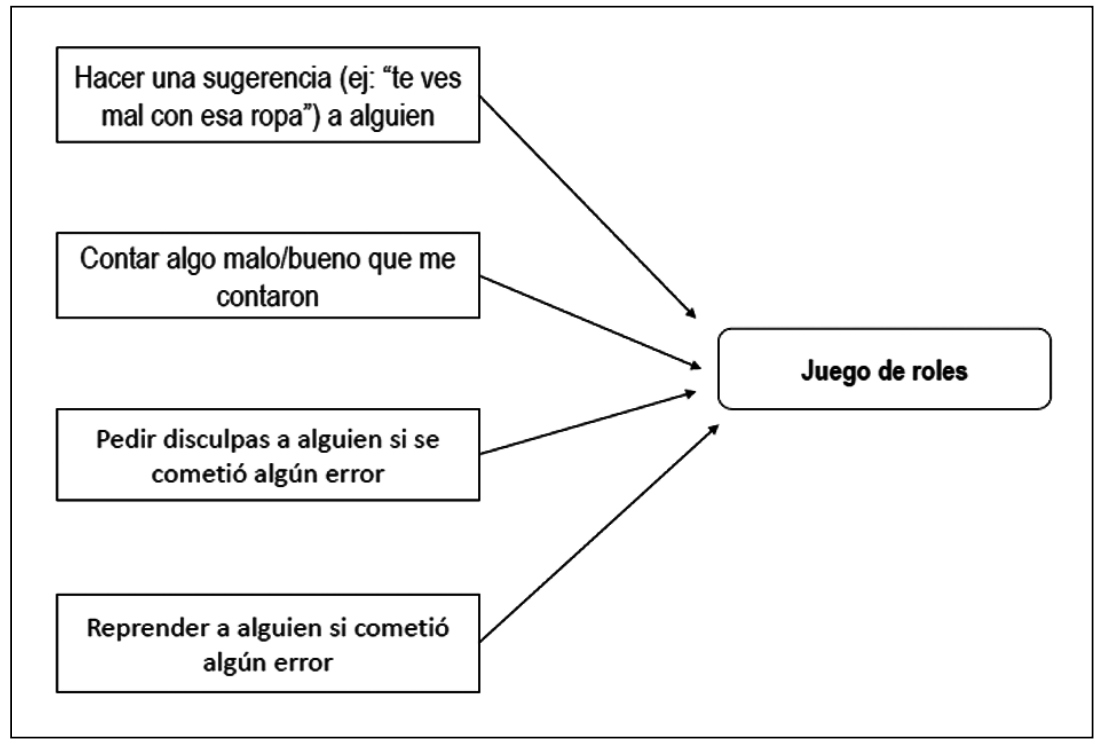

Figura 6. Tareas pragmalingüísticas para mejorar el desempeño comunicativo. 
Tabla 2. Resultados de Eyes test inicio y finalización del CONECTA-2

\begin{tabular}{|cccccc|}
\hline n & Sexo & Edad & Eyes 1 & Eyes 2 & $\begin{array}{c}\text { Incremento } \\
\text { pje Eyes test }\end{array}$ \\
\hline PEE-1 & M & 19 & 18 & 23 & 5 \\
PEE-2 & M & 18 & 17 & 22 & 5 \\
PEE-3 & F & 18 & 19 & 19 & 0 \\
PEE-4 & M & 18 & 20 & 22 & 2 \\
PEE-5 & M & 24 & 20 & 24 & 4 \\
PEE-6 & M & 19 & 20 & 23 & 3 \\
PEE-7 & M & 21 & 19 & 25 & 6 \\
PEE-8 & F & 24 & 20 & 24 & 4 \\
\hline PEE-9 & F & 24 & 19 & 25 & 6 \\
\hline PEE-10 & M & 24 & 19 & 23 & 4 \\
\hline
\end{tabular}

de 5 y 6 puntos en el segundo Eyes test, en otros tres pacientes se logró un incremento de 4 puntos y en dos de ellos solo varió en 2 y 3 puntos. Estos resultados son relevantes para la toma de decisiones clínicas y terapéuticas con pacientes de primer episodio. A continuación, en la Tabla 2, se resumen los resultados de la evaluación inicial y de salida del taller.

En cuanto a los resultados de la aplicación del screening LEPSI, podemos señalar que a nivel general, los participantes que presentaban un nivel de rendimiento pragmalingüístico bajo o intermedio, experimentaron una mejoría en la segunda evaluación o mantuvieron el mismo nivel de desempeño que en la primera. En estricto orden descendente, los ítems en los que mejoraron los participantes son los siguientes; el de aspectos paraverbales, que se incrementó hasta en un $70 \%$ en la segunda evaluación, aumento que puede estar motivado por el entrenamiento constante en estrategias paraverbales que fueron acompañando gran parte de las actividades de los módulos a lo largo del taller. Luego es destacable el incremento en los ítems de teoría de la mente y cognición social y el de coherencia interaccional, en ambos se alcanzó un $60 \%$ en la segunda evaluación, en este caso, el aumento se vincula con el tipo de tareas que se desarrollaron en estos módulos, las cuales consistieron en centrarse en las necesidades comunicativas del otro. En el ítem de organización pragma-lingüística global, el 50\% de los participantes mejoró su desempeño en la segunda aplicación del screening, en este ítem se valoró especialmente la eficacia en el manejo de los objetivos discursivos que se plantearon en el curso de la interacción. En el ítem de coherencia en la organización estructural, el $40 \%$ de los participantes mejoraron su nivel de desempeño, nos parece una cifra relevante puesto que este ítem se compone de 7 elementos necesarios para estabilizar la coherencia del tema y la estructura del discurso, tales como: macroestructuras; gestión del tema; desarrollo de tópicos, entre otros recursos que requieren una complejidad mayor. A continuación, en la Tabla 3, reseñamos los puntajes obtenidos en ambos talleres.

\section{Discusión y Conclusiones}

A través de la comunicación, establecemos relaciones con los demás y las mantenemos. Por tanto, aquellos individuos que se ven afectados por alguna patología en la que no pueden negociar el significado o adecuarse apropiadamente a los contextos comunicativos en que deben desenvolverse ven deteriorada e incluso ausente su imagen social (Goffman, 1970). Junto con la imagen social o face descrito por Goffman el lenguaje determina el rol que deben atender los hablantes para determinar, evaluar y comprender indicios de claves sociales que les permitan una participación dinámica y eficiente en el medio en el que están (Couture, 2006).

Existen mecanismos y estrategias que los hablantes adquieren en el transcurso de su participación en una comunidad social determinada, esta habilidad se convierte en una manifestación social que cataliza la inclusión o exclusión de los individuos. La pérdida o deterioro progresivo de estas habilidades supone un impedimento insalvable para mantener el estatus psicosocial vinculante dentro del medio al que se pertenece. 
Tabla 3. Evaluación inicial y final con screening LEPSI

\begin{tabular}{|c|c|c|c|c|c|c|c|c|c|c|c|c|c|c|c|}
\hline \multirow{2}{*}{$\begin{array}{l}\text { Cluster } \\
\text { Test-retest }\end{array}$} & \multicolumn{3}{|c|}{$\begin{array}{l}\text { Org. pragmalin- } \\
\text { güística global }\end{array}$} & \multicolumn{3}{|c|}{$\begin{array}{l}\text { Org. estructural } \\
\text { del discurso }\end{array}$} & \multicolumn{3}{|c|}{$\begin{array}{l}\text { Teoría de la mente } \\
\text { y cognición social }\end{array}$} & \multicolumn{3}{|c|}{$\begin{array}{l}\text { Coherencia } \\
\text { interaccional }\end{array}$} & \multicolumn{3}{|c|}{$\begin{array}{c}\text { Aspectos } \\
\text { paraverbales }\end{array}$} \\
\hline & $\begin{array}{l}\text { LEPSI } \\
\text { I }\end{array}$ & $\begin{array}{l}\text { LEPSI } \\
\text { II }\end{array}$ & Res & LEPSI & $\begin{array}{l}\text { I LEPSI } \\
\text { II }\end{array}$ & Res & LEPSI 1 & $\begin{array}{c}\text { I LEPSI } \\
\text { II }\end{array}$ & Res & LEPSI & $\begin{array}{l}\text { LEPSI } \\
\text { II }\end{array}$ & Res & LEPSI I & $\begin{array}{l}\text { LEPSI } \\
\text { II }\end{array}$ & Res \\
\hline PEE1 & 3 & 3 & $\rightarrow$ & 14 & 14 & $\rightarrow$ & 10 & 12 & $\uparrow$ & 20 & 30 & $\uparrow$ & 5 & 10 & $\uparrow$ \\
\hline PEE2 & 3 & 3 & $\rightarrow$ & 14 & 14 & $\rightarrow$ & 10 & 12 & $\uparrow$ & 20 & 30 & $\uparrow$ & 5 & 10 & $\uparrow$ \\
\hline PEE3 & 2 & 2 & $\rightarrow$ & 14 & 14 & $\rightarrow$ & 4 & 10 & $\uparrow$ & 10 & 20 & $\uparrow$ & 5 & 5 & $\rightarrow$ \\
\hline PEE4 & 2 & 3 & $\uparrow$ & 14 & 14 & $\rightarrow$ & 10 & 10 & $\rightarrow$ & 20 & 30 & $\uparrow$ & 10 & 10 & $\rightarrow$ \\
\hline PEE5 & 2 & 3 & $\uparrow$ & 14 & 21 & $\uparrow$ & 4 & 10 & $\uparrow$ & 20 & 20 & $\rightarrow$ & 10 & 15 & $\uparrow$ \\
\hline PEE6 & 1 & 2 & $\uparrow$ & 7 & 14 & $\uparrow$ & 4 & 10 & $\uparrow$ & 20 & 20 & $\rightarrow$ & 5 & 10 & $\uparrow$ \\
\hline PEE7 & 2 & 3 & $\uparrow$ & 14 & 14 & $\rightarrow$ & 10 & 10 & $\rightarrow$ & 20 & 30 & $\uparrow$ & 5 & 5 & $\rightarrow$ \\
\hline PEE8 & 3 & 3 & $\rightarrow$ & 14 & 21 & $\uparrow$ & 12 & 12 & $\rightarrow$ & 30 & 30 & $\rightarrow$ & 10 & 15 & $\uparrow$ \\
\hline PEE9 & 2 & 3 & $\uparrow$ & 14 & 21 & $\uparrow$ & 4 & 10 & $\uparrow$ & 20 & 30 & $\uparrow$ & 10 & 15 & $\uparrow$ \\
\hline PEE10 & 2 & 2 & $\rightarrow$ & 14 & 14 & $\rightarrow$ & 10 & 10 & $\rightarrow$ & 20 & 20 & $\rightarrow$ & 5 & 10 & $\uparrow$ \\
\hline Ptjes totales & \multicolumn{3}{|c|}{1 Îtem $=3 p$} & \multicolumn{3}{|c|}{7 Ítem $=21 p$} & \multicolumn{3}{|c|}{4 Ítem $=12 p$} & \multicolumn{3}{|c|}{10 Ítem = 30p } & \multicolumn{3}{|c|}{5 Ítem $=15 p$} \\
\hline
\end{tabular}

Esta experiencia piloto, lleva a plantearnos el desafío de implementar nuevas líneas de investigación y de iniciar, de manera sistemática, dispositivos terapéuticos de entrenamiento en cognición social y habilidades pragmalingüísticas, dado el déficit presentado por personas con esta patología, y por su relevancia en la rehabilitación de su adaptación interpersonal.

\section{Resumen}

Introducción: En el presente trabajo, nos proponemos describir una experiencia terapéutica piloto con un programa desarrollado por el equipo, al que denominamos CONECTA-2, un plan de entrenamiento de cognición social y habilidades comunicativas para personas con diagnóstico de esquizofrenia. Nuestro objetivo fue establecer si un dispositivo clínico terapéutico teóricopráctico, de entrenamiento grupal en funciones ejecutivas, de procesos de cognición social y lenguaje, puede mejorar la eficacia comunicativa en personas con diagnóstico de esquizofrenia. Método: Participaron 10 personas entre 18 y 24 años, 7 hombres y 3 mujeres, con diagnóstico de esquizofrenia desde hace menos de tres años al momento de efectuar el taller. Se realizó una evaluación previa y posterior de atribución emocional y eficacia comunicativa. Se realizaron 24 sesiones grupales, de entrenamiento en funciones ejecutivas, cognición social y habilidades pragmático-comunicativas. Resultados: Se verificaron cambios positivos tanto en atribución emocional como en la eficacia comunicativa en 9 de los 10 participantes. Conclusiones: Los resultados de esta experiencia piloto, nos plantean un desafío en términos de desarrollar planes de intervención clínica, que permitan rehabilitar aquellas habilidades, en pos de lograr una mejor adaptación e inserción social de personas con esquizofrenia.

Palabras clave: Esquizofrenia, lenguaje, cognición, comunicación. 


\section{Referencias bibliográficas}

1. Adolphs R. The neurobiology of social cognition. Curr Opin Neurobiol 2001; 11:231-9.

2. Baron-Cohen S. The theory of mind deficit in autism: How specific is it? Br J Dev Psychol 1991; 9: 301-14.

3. Baron-Cohen S, Wheelwright S, Hill J, Raste Y, Plumb I. The 'Reading the mind in the eyes' test revised version: A study with normal adults, and adults with Asperger Syndrome or High-Functioning autism. J Child Psychol Psychiatry 2001; 42: 241-52.

4. Brothers L. The social brain: a project for integrating primate behavior and neurophysiology in a new domain. Concepts Neurosci 1990; 1:27-61.

5. Brothers L, Ring B. A neuroethological framework for the representation of minds. J Cogn Neurosci 1992; 4 (2): 107-18.

6. Burin D. Evaluación Neuropsicológica en Adultos, Paidós, Buenos Aires Argentina. 2008.

7. Couture SM, Penn DL, Roberts DL. The Functional Significance of Social Cognition in Schizophrenia: A Review. Schizophr Bull 2006; 32: 44-63.

8. Fett AK, Viechtbauer W, Domínguez MD, Penn DL, van Os J, Krabbendam L. The relationship between neurocognition and social cognition with functional outcomes in schizophrenia: a metaanalysis. Neurosci Biobehav 2011; 35 (3): 573-88.

9. Figueroa A, Oyarzún S, Sepúlveda P. Rol de la pragmática y la Cognición Social en la esquizofrenia de primer episodio, en Figueroa A y Durán E. (coord.) Psicolingüística Clínica aplicada a las enfermedades mentales. 2011; Cap. 2, pp. 39-56.
Lingua \& Psyké No 1 . Corporación Chilena de la Esquizofrenia, CORES y Universidad de Chile.

10. Grice P. "Presupposition and Coversational Implicature.” En: Cole 1981, págs 183-98.

11. Leslie A, Frith U. Prospects for a cognitive neuropsychology of autism: Hobson`s choice. Psychological Review 1990; 97 (1): 122-31.

12. Pijnenborg GH, Withaar FK, Evans JJ, Van den Bosch RJ, Timmerman ME, Brouwer WH. The predictive value of measures of social cognition for community functioning in schizophrenia: implications for neuropsychological assessment. J Int Neuropsychol Soc 2009; 15: 239-47.

13. Roder V, Medalia A (eds). Neurocognition and Social Cognition in Schizophrenia Patients. Basic Concepts and Treatment. Key Issues Ment Health Basel, Karger 2010; 177: 23-36.

14. Schmidt SJ, Mueller DR, Roder V. Social Cognition as a Mediator Variable Between Neurocognition and Functional Outcome in Schizophrenia: Empirical Review and New Results by Structural Equation Modeling. Schizophr Bull 2011; 37 Supl 2: 41-54.

15. Sperber D, Wilson D. La teoría de la relevancia. Revista de Investigación Lingüística 2004; VII: 237-86.

16. Stone VE, Baron-Cohen S, Knight RT. Frontal lobe contributions to theory of mind. J Cogn Neurosci 1998; 10: 640-56.

17. Yirmiya N, Sigman MD, Kasari C, Mundy P. Empathy and cognition in high-functioning children with autism. Child Development 1992; 63: 150-60.

18. Wilson D, Sperber D. Meaning and Relevance, Nueva York: Cambridge University Press. 2012.

Correspondencia:

Alicia Figueroa Barra

Email: aliciafigueroa@med.uchile.cl 